Instituto Internacional de Investigación y Desarrollo Tecnológico Educativo INDTEC, C.A.

DOI: https://doi.org/10.29394/Scientific.issn.2542-2987.2020.5.15.12.247-268

OAI-PMH: http://www.indteca.com/ojs/index.php/Revista Scientific/oai

Artículo Original / Original Article

\title{
Cultura de datos y mejora escolar: toma de decisiones educativas basadas en evidencias
}

\author{
Autoras: Melvis Lissety González Acosta \\ Universidad Nacional de Educación, UNAE \\ melvis.gonzalez@unae.edu.ec \\ Azogues, Ecuador \\ https://orcid.org/0000-0002-5672-6543 \\ Diana Isabel Rodríguez Rodríguez \\ Universidad Nacional de Educación, UNAE \\ diana.rodriguez@unae.edu.ec \\ Azogues, Ecuador \\ https://orcid.org/0000-0003-4406-9555
}

\section{Resumen}

La presente investigación tuvo como objetivo aprovechar el potencial del récord académico de los estudiantes para guiar la práctica docente al aumento del éxito educativo. Plantea como hipótesis que el análisis de la información contenida en el récord académico contribuye a transformar los procesos de enseñanza/aprendizaje, en cuatro niveles: descriptivo (qué ocurre); diagnóstico (por qué ocurre), predictivo (qué puede suceder) y prescriptivo (cómo se puede mejorar). Se trató de un estudio prospectivo en el cual se recolectaron datos históricos: notas de la asignatura de matemáticas del récord académico de un grupo de 15 estudiantes que han permanecido juntos durante cinco cursos escolares, en un periodo que va desde septiembre del año 2014 hasta julio del año 2019. Los resultados muestran que, en los primeros cinco años de estudio de las matemáticas, las calificaciones tienden a decrecer, y cuando se manifiesta un ascenso mínimo es por debajo del punto de partida. El bajo rendimiento académico a causa de las dificultades para el aprendizaje de la matemática está vinculado a posteriores deserciones en los primeros ciclos de la universidad, y constituye un problema actual de alcance nacional e internacional, que comparten un número considerable de instituciones educativas.

Palabras clave: enseñanza y formación; aprendizaje a través de la experiencia; estudiante de primaria; profesionales de la educación.

Cómo citar este artículo:

González, M., \& Rodríguez, D. (2020). Cultura de datos y mejora escolar: toma de decisiones educativas basadas en evidencias. Revista Scientific, 5(15), 247-268, e-ISSN: 2542-2987. Recuperado de: https://doi.org/10.29394/Scientific.issn.2542-2987.2020.5.15.12.247-268

Fecha de Recepción: 23-09-2019
Fecha de Aceptación: 21-12-2019
Fecha de Publicación: 05-02-2020 


\title{
Data culture and school improvement: evidence-based educational decision making
}

\begin{abstract}
This research aimed to harness the potential of the student's academic record to guide teaching practice to increase educational success. It is hypothesized that the analysis of the information contained in the academic record contributes to transforming the teaching / learning processes into four levels: descriptive (what happens); diagnosis (why it occurs), predictive (what can happen) and prescriptive (how it can be improved). It was a prospective study in which historical data were collected: mathematics subject from the academic record of a group of 15 students who have remained together for five school years, in a period from September 2014 to July of the year 2019. The results show that, in the first five years of study of mathematics, grades tend to decrease, and when a minimum rise is manifested, it is below the starting point. The low academic performance due to the difficulties for learning mathematics is linked to subsequent defections in the first cycles of the university, and constitutes a current problem of national and international scope, which share a considerable number of educational institutions.
\end{abstract}

Keywords: teaching and training; experiential learning; primary school student; educational population.

\section{How to cite this article:}

González, M., \& Rodríguez, D. (2020). Data culture and school improvement: evidence-based educational decision making. Revista Scientific, 5(15), 247-268, e-ISSN: 2542-2987. Recovered from: https://doi.org/10.29394/Scientific.issn.2542-2987.2020.5.15.12.247-268

Date Received: 23-09-2019
Date Acceptance:

21-12-2019
Date Publication: 05-02-2020 


\section{Introducción}

El uso efectivo de los datos en la era digital resulta fundamental para mejorar casi cualquier proceso. Nos referimos a datos como al conjunto de información que sobre una determinada unidad o entidad se ha conseguido recopilar, y que pueden ser utilizados por varias personas para alcanzar fines relacionados con la toma de decisiones.

Si consideramos a los estudiantes como parte de una entidad concreta, podemos decir que, durante la vida académica de todos ellos, se ha conseguido recopilar abundante información. El récord académico o historial que corresponde a cada miembro del conjunto de datos, por sí solo no dice mucho; pero esos datos analizados desde la complejidad, se convierten en una fuente de información pertinente que puede marcar la diferencia. Sin embargo, a pesar de su proliferación, la información que contienen estos conjuntos de datos, sigue siendo subutilizada por la comunidad educativa para aumentar el desempeño de los estudiantes.

Los sistemas educacionales y miembros de la comunidad educativa que tienen la responsabilidad de tomar decisiones en el proceso de enseñanzaaprendizaje, precisan planificar un uso más eficiente de los datos (evaluar problemas, desafiar creencias, exponer diferentes hipótesis y/o plantear preguntas más focalizadas) como contribución a la transformación de las prácticas educativas y mejoramiento del desempeño escolar. Tal determinación, favorece acciones más contextualizadas, tomando en consideración que la visualización y valor contenido en los datos contribuye a despejar interrogantes sobre los cambios requeridos para suscitar el mejoramiento escolar.

Si bien las posibilidades que ofrece el análisis de datos en los entornos educativos, favorecen el cambio de perspectiva sobre la toma de decisiones, continúa siendo endeble su reconocimiento, análisis e implementación. Respecto a la incorporación del estudio de datos como paradigma 
transformador en la sociedad digital, el experto en macrodatos y analíticas de aprendizaje, Julià Minguillón, considera según Carrillo (2016): que "es necesario incorporar una cultura de datos en las instituciones educativas para tomar decisiones basadas en evidencias, no solo en suposiciones, formas de hacer o experiencias que no hayan sido validadas formalmente" (pág. 1).

Tomando como punto de partida este enfoque, se plantea como hipótesis que el análisis de la información contenida en el récord académico de los estudiantes, contribuye a transformar los procesos de enseñanzaaprendizaje, en cuatro niveles: descriptivo (qué ocurre); diagnóstico (por qué ocurre), predictivo (qué puede suceder) y prescriptivo (cómo se puede mejorar). La idea contenida en la hipótesis ha sido formulada por Salvador Rojas basándose en que el tratamiento y análisis de los datos generados en el contexto académico, permite entender cómo se produce el aprendizaje, qué factores pueden influir positiva o negativamente y qué se podría hacer para mejorar, señala Hernández (2018): que esto se logra a partir de la interacción con el contenido del dato y la colaboración entre los miembros de la comunidad educativa (pág. 1).

Cuando un conjunto de datos se examina a la luz de una teoría, se puede apreciar la información contenida en los mismos. El objetivo de la siguiente investigación fue aprovechar el potencial del récord académico de los estudiantes para guiar la práctica docente al aumento del éxito educativo.

\section{Antecedentes investigativos}

El tema del análisis de datos y el valor de la evidencia en la toma de decisiones en temas educativos, ha ganado relevancia en lo que transcurre del Siglo XXI. La investigación de Parra y Matus (2016): muestra orientaciones a la política educativa con miras a potenciar el uso contextualizado, pertinente y oportuno de datos en la gestión de las escuelas (págs. 4-7). Otras propuestas como la de Dougherty (2015a): que emplean los datos para evaluar la calidad 
de la enseñanza-aprendizaje, entrenamiento y supervisión a los maestros, y para guiar las decisiones administrativas (pág. 1). En cada caso se trata de una respuesta a la necesidad de contar con estrategias que apoyen las trayectorias educativas de los estudiantes y la mejora de la escuela, presentada por los autores Bernhardt (2000); Codding y Rothman (1999).

Para Coughlin (2014): la teoría sobre la necesidad de un uso más efectivo de los datos, contrasta con la necesidad de ayuda por parte de colegios y universidades para hacer un buen uso de los resultados de las evaluaciones para mejorar la enseñanza y el aprendizaje (pág. 1). Mientras que Millett, Payne, Dwyer, Stickler y Alexiou (2008): exponen que los docentes se enfrentan a problemas y matices inherentes a la evaluación del aprendizaje de los estudiantes, sin ser parte de una cultura centrada en la evidencia para la rendición de cuentas por los resultados de aprendizaje de los estudiantes (pág. 1).

La investigación de Dougherty (2015b): muestra que autoridades educativas utilizaron la evaluación y otros tipos de datos para evaluar la calidad de la enseñanza y el aprendizaje, para entrenar y supervisar a los maestros, y para guiar las decisiones administrativas; además de apoyar el uso de datos por parte de los maestros (pág. 1). En tanto, la disponibilidad de los datos es una limitante de esta investigación. Al no estar en presencia de una cultura de uso de datos, por pequeña que sea la muestra, se dificulta obtenerla. A esto agréguese la procedencia del investigador; si no deriva de la Institución, recae la sospecha en el interés de la investigación y existe la tendencia a actuar con desconfianza sobre la conveniencia. En este sentido, es frecuente recibir información aparentemente objetiva que, a pesar de ello, resulta dudosa por algún motivo (por ejemplo, porque es tan favorable que no parece creíble).

Matemáticas fue considerada como referente de análisis, tomando en consideración el significado que tiene para el desarrollo intelectual de las 
personas, el hecho de ayudar a razonar ordenadamente y a tener una mente preparada para el pensamiento, la creatividad, la crítica y la abstracción. Lo cual contrasta con las creencias epistemológicas sobre esta materia, que ocasionan rechazo por parte de los estudiantes, con consecuencias desfavorables que se arrastran a lo largo de la vida. Y que según Boaler (2016a): están relacionadas y "lo que creemos sobre nosotros mismos tiene un gran impacto sobre lo que aprendemos y sobre cómo lo aprendemos" (pág. 1); de modo que la mayor parte de personas que piensan que son malos en matemáticas, lo son, como resultado de una profecía autocumplida. No es que rechazan las matemáticas porque se les dan mal, sino que se les dan mal porque las rechazan.

La materia de matemáticas dentro del currículo nacional ecuatoriano cumple un rol decisivo tanto en su crecimiento como asignatura, como en el perfil de salida del bachiller. El conocimiento matemático se emplea en dicha materia y se convierte en cualidad aplicativa en forma interdisciplinaria. Es indispensable considerar la relevancia de la materia de estudio y sus expectativas de mejora en pro de una educación de calidad.

\section{Metodología (materiales y métodos)}

Se trató de un estudio prospectivo en el cual se recolectaron datos históricos: notas de la asignatura de matemáticas del récord académico de un grupo de 15 estudiantes. Se dividen en ocho (8) niñas y siete (7) niños, que han permanecido juntos durante cinco cursos escolares: desde segundo hasta sexto grado de enseñanza básica, en un periodo que va desde septiembre del año 2014 hasta julio del año 2019.

La permanencia, entendida como el mantenimiento de los estudiantes en el mismo grupo a través del tiempo sin variar sus características, fue una cualidad subjetiva intrínseca en el análisis, por considerar parte del mejoramiento escolar tanto el desempeño individual del estudiante, como su 
desempeño colectivo y la manera como es influido por el grupo de pares, el docente y el propio contexto educativo, caracterizado por la cooperación entre miembros de la comunidad y posibilidades de actuación. El desempeño colectivo constituyó el elemento de análisis; en este caso, si se toma en consideración la disposición frente a determinadas situaciones, tanto de estudiantes como docentes.

El diseño de investigación se sustentó en el paradigma mixto con un enfoque fenomenológico a través de un estudio de caso y orientado a la explicación, comprensión y contribución a la transformación de la educación basado en evidencias. El enfoque mixto involucró la combinando de una metodología cuantitativa con una cualitativa, para aumentar la validez y consistencia de los hallazgos. El modelo fenomenológico resultó particularmente útil para el análisis sobre los niveles descriptivo (qué ocurre) y diagnóstico (por qué ocurre), y posterior interpretación sobre los niveles predictivo (qué puede suceder) y prescriptivo (cómo se puede mejorar).

El caso de estudio en particular se reportó desde la escuela privada de enseñanza básica "Eugenio Espejo" localizada en el cantón Ambato, provincia Tungurahua, Ecuador. Si bien el tamaño de la muestra es reducido, permitió abordar de manera colaborativa el análisis de un fenómeno (desempeño académico) exactamente en el lugar y condiciones donde el mismo se despliega, y contribuir a desarrollar una cultura de uso de datos para la toma de decisiones basadas en evidencias.

Las técnicas utilizadas para la recolección de la información fueron: la observación etnográfica, el análisis documental y el diálogo entre docentepadres-estudiantes-autoridades educativas. Para la interpretación de los datos cuantitativos, se empleó el conocimiento del grupo de estudio por parte de docentes, padres de familia, los propios estudiantes y las autoridades educativas. 


\section{Resultados (análisis e interpretación de los resultados)}

La calificación o nota utilizada para evaluar y categorizar el rendimiento escolar, proporciona más que solo un destino. Por ejemplo, además de marcar qué tan distante están los estudiantes del mínimo o máximo aprobatorio, ayuda a interpretar y actuar sobre lo que aprenden.

En caso de los docentes, proporciona una guía que les informe si están moviéndose en la dirección correcta u ofrecer una retroalimentación interactiva y recursiva para realizar ajustes de mitad de curso. Los docentes que adecuan sus lecciones al nivel de desarrollo apropiado de sus estudiantes, tienen más probabilidades de proporcionar aprendizaje significativo.

Si bien profesores y teóricos consideran que la evaluación tradicional por calificaciones es un método obsoleto para la educación del Siglo XXI, es un método que se sigue aplicando. De allí que se pretenda aprovechar la data para analizar el estado de la cuestión sobre qué estamos dejando de ver y cuánto podemos transformar ese panorama.

\section{1. ¿Qué ocurre?}

En el gráfico 1, se visualizan las calificaciones de matemáticas del grupo de 15 estudiantes que conforman la muestra y que han cursado juntos desde segundo hasta sexto grado de enseñanza básica. Lo que sorprendería a cualquier docente es que, en los primeros cinco años de estudio de las matemáticas, las calificaciones tienden a decrecer de manera significativa, y cuando se manifiesta un ascenso mínimo es por debajo del punto de partida.

Se aprecia un crecimiento en los inicios de la asignatura, en ambos sexos; comprensible por cuanto es un aprendizaje básico que tiene que progresar. Las niñas mantienen un nivel más elevado en las calificaciones, lo que contrasta con la idea de que los niños son mejores en matemáticas, y da cuenta de que los estereotipos sobre las niñas y las matemáticas no están basados en investigaciones. 
Gráfico 1. Promedio de calificaciones de niñas y niños en matemáticas.

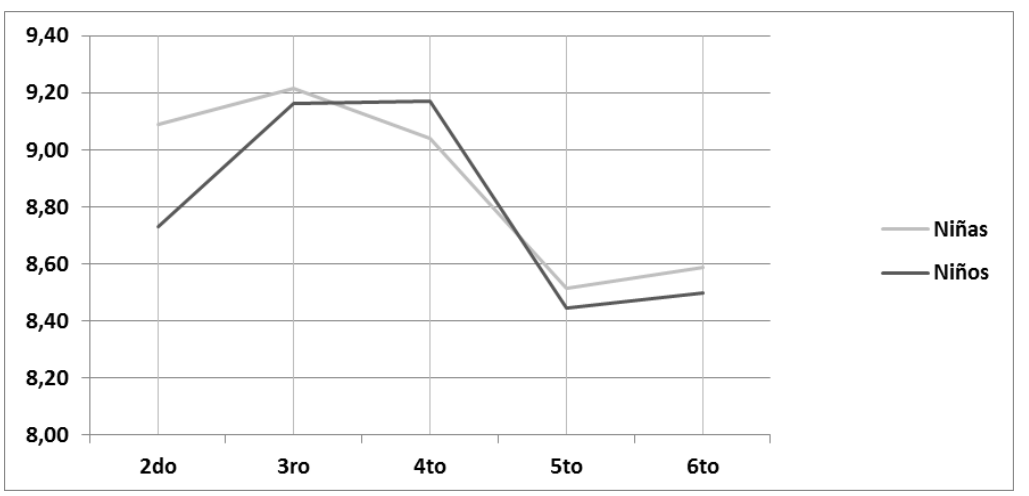

Fuente: Las Autoras (2019).

Un estudio realizado en China por Zhou (2012): sugiere que las habilidades verbales superiores de las niñas podrían explicar la ventaja matemática, ya que contar es algo verbal, la tabla de multiplicar se memoriza verbalmente, y cuando se calcula, los resultados intermedios se mantienen en la memoria como palabras (pág. 1). Esto podría explicar que más capacidad lingüística supone un procesamiento verbal más eficiente en la matemática.

Según Boaler (2016b): para que las matemáticas sean del agrado de las niñas debe cambiarse la forma en que se enseñan, basada fundamentalmente en la representación de un proceso abstracto (pág. 1). Su idea se basa en la necesidad que tienen las niñas de establecer conexiones: entre la propia disciplina, y de esta con la vida real. Mantener el interés de las niñas en las matemáticas contribuye a empoderarlas en los campos de ciencia, tecnología e ingeniería y les ofrece mayores oportunidades en una sociedad altamente competitiva y de profundas asimetrías sociales influenciadas por el género.

En el cuarto año el promedio de calificaciones decreció en las niñas y se incrementó una décima en los niños. Para el quinto año continuó el descenso, ahora en ambos géneros. El análisis de esta figura hace recapacitar a los docentes y autoridades de la unidad educativa que las causas se deban 
al aumento del horizonte de competencias generales y destrezas vinculados a diferentes niveles de enseñanza. Evidencia la transición de los alcances durante el proceso de aprendizaje de cuarto grado, que se corresponde con básica elemental a quinto grado, perteneciente a básica media. En tal caso, persisten lagunas de conocimientos que dificultan la apropiación de nuevos elementos para el razonamiento, conexión y resolución de problemas.

El objetivo de la investigación y los datos que se analizan, no posibilitan un estudio sobre el cómo el género contribuye a la diferencia; pero recalca la necesidad de proveer a todos los estudiantes igualdad de oportunidades, según sus necesidades, para aprehender las matemáticas y hacer uso de la misma para solucionar problemas de la vida cotidiana.

Grosso modo se aprecia que el aprendizaje es un proceso que puede tomar diferentes formas. La trayectoria está vinculada a factores como: relaciones interpersonales, actitudes, conocimientos previos, experiencias vividas, metodología del docente, recursos empleados, hasta modificaciones en los planes de estudio.

La investigación realizada por Boaler (2016c): manifiesta que la trayectoria que pueda seguir el proceso de enseñanza-aprendizaje de las matemáticas, genere un ambiente de competencia negativa que provoque sentimiento de rechazo hacia la materia. Por ejemplo, un gran problema es que la matemática se ha establecido como una materia de velocidad (pág. 1). Las pruebas de velocidad contaminan de ansiedad los salones de clases y crean una impresión de que la velocidad es lo más importante. Esto afecta a quienes son lentos o a quienes se toman su tiempo para pensar, entre ellos las niñas.

En el gráfico 2, se observa que, en cuatro de los cinco años de estudios, los resultados del segundo quimestre son inferiores al primero, en correspondencia con la tendencia a tener un buen comienzo y en el transcurso del quimestre ir ganando frustración como una consecuencia negativa que 
puede provocar daños y afectar la motivación. La disminución de la motivación está asociada a la aplicación de metodologías tradicionalistas por parte del docente como el aprendizaje memorístico, la oralidad en la comprensión de los ejercicios matemáticos y la falta de contextualización que limita el proceso de enseñanza y aprendizaje.

Gráfico 2. Promedio de calificaciones por quimestres (QUIM) y años de estudio.

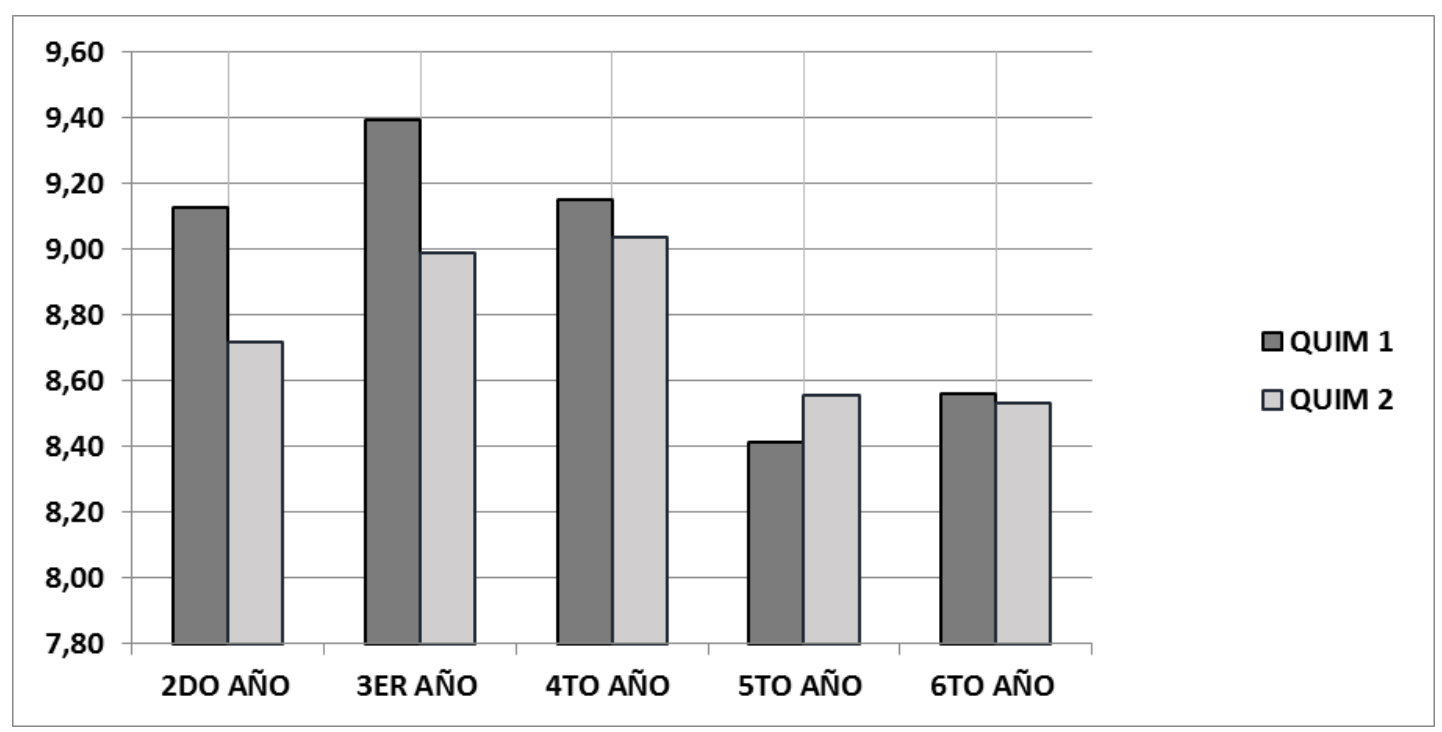

Fuente: Las Autoras (2019).

\section{2. ¿Por qué ocurre?}

Cuando se les preguntaron a los docentes las causas para este comportamiento comentaron que el dominio de la operación aritmética de multiplicación, retardó más de lo planificado, la comprensión de las operaciones de división; generando dificultades para los estudiantes. En términos de adquisición de competencias, estos estudiantes hasta cuarto grado, en correspondencia con el plan de estudio, venían practicando la suma, la resta, la multiplicación, los problemas matemáticos de lógica y la división en papel; y el quinto grado les exigía incrementar las fracciones y los decimales. 
En respuesta a la demanda estudiantil, que no se correspondía al aumento previsto de la complejidad en la asimilación de contenidos, los docentes se aproximaron a la práctica de ejercicios de cálculo mental, para facilitar el proceso de enseñanza-aprendizaje.

Existe un componente externo que incide en el desempeño estudiantil, vinculado a las reformas curriculares. El objetivo de los ajustes, si bien responde a un análisis en el que participaron docentes de todo país: 1). Estar enfocado en el desarrollo del pensamiento crítico y reflexivo que contribuye a la resolución de problemas de la vida cotidiana; y 2). Vinculado a la integración con el subnivel superior de educación general básica y nivel de bachillerato; genera problemas por el tiempo asignado a su cumplimiento, así como problema de conexión con los subniveles inferiores.

Siguiendo a Navarro-Aburto, Arriagada, Osse-Bustingorry y BurgosVidela (2016): las adaptaciones curriculares son "el instrumento fundamental para conseguir la individualización de la enseñanza, por cuanto son modificaciones que se realizan en la programación curricular común para entender las diferencias individuales del alumnado" (pág. 2); pero implican dificultades tanto para docentes como estudiantes; tal es el caso de la introducción de temas, en quinto grado, que anteriormente se impartían en sexto curso. Esta fusión de contenidos en los diferentes subniveles de enseñanza, todavía se estaban perfeccionando dos años después de la entrada en vigor de la adaptación curricular del año 2016.

Al preguntarle a los estudiantes, la primera reacción fue la sorpresa. Cada uno tenía una percepción sobre si se consideraba bueno o malo en matemáticas, pero no sabían si estaban mejor o peor que años anteriores. No sabían cuál era el comportamiento como grupo, ni tenían ideas de los resultados en niñas y niños. Lo que sucede se puede sintetizar en tres consideraciones fundamentales: 1). El nivel de abstracción de la asignatura que va de menos a más y paralelo al carácter acumulativo, hace que los niños 
se desorienten respecto a su desempeño; 2). El cambio de docente, contribuye a que los estudiantes se aproximen a las matemáticas como algo nuevo: resulta que lo que resolvía un año de una forma al año siguiente se resuelve de otra, solo por cambiar de año y/o profesor; y 3). Les han cambiado las formas de analizar un ejercicio, sin explicarlos por qué es posible hacerlo de diferentes maneras; por lo cual los estudiantes no identifican que tienen que acudir a determinado conocimiento en el nuevo contexto en el que se le plantea un problema.

Se ha considerado de manera general que la clave para entender el por qué ocurre está en cómo se enseñan las matemáticas. Si en vez de identificar los errores, pensar, discutir, tomar decisiones y después dar cuenta de por qué decidió de la forma que lo hizo, el estudiante reproduce lo que le enseñaron, tal y como se lo enseñaron. La prioridad recae en los procedimientos para la enseñanza-aprendizaje de la materia.

El otro camino sería que el peso de la valoración recayera en la trayectoria de los aprendizajes: en qué contenidos y de qué modo se han aprehendido, sin perder de vista a los estudiantes. Estos, deben tener confianza en que no solo tienen que resolver problemas, además pueden crearlos o tomar decisiones sobre la variedad de formas de pensar en el problema. Por ejemplo, ilustra Boaler (2016d):

Si un profesor les pide a sus estudiantes que encuentren el área de un rectángulo de $8 \times 3$, en ese caso, el cálculo tiene una sola respuesta. Pero si el profesor ve el problema más conceptual y quiere volver el ejercicio más difícil, les puede preguntar a sus alumnos cuántos rectángulos son necesarios para tener un área de 24. Eso los obliga a pensar en las dimensiones y cómo la longitud y la anchura se relacionan entre sí (pág. 1).

El tipo de dato recopilado en esta investigación muestra el desempeño en matemáticas del grupo de estudiantes, a lo largo de su contacto con la 
materia. Comprobar la capacidad que ofrecen las matemáticas de formular, emplear, interpretar y explicar cuestiones de la vida cotidiana, que enriquecen la experiencia individual, requeriría de otro tipo de estudio, más preciso, que no se adscribe a los fines de esta investigación.

\section{3. ¿Qué puede suceder?}

Las matemáticas pueden resultar frustrantes y provocar actitudes negativas hacia ellas. El bajo rendimiento académico a causa de las dificultades para el aprendizaje de la matemática está vinculado a las posteriores deserciones en los primeros ciclos de la universidad, y constituye un problema actual de alcance nacional e internacional, que comparten un número considerable de instituciones educativas.

Sería prematuro e infundado decir que el grupo de estudio vaya camino al fracaso, porque sus resultados numéricos demuestran ser favorables. Lo percibido tiene causas relativas al:

1). Desarrollo cognitivo propio de la edad de los estudiantes como la inmadurez y la limitada capacidad para comprender la lógica, el simbolismo y la abstracción de las matemáticas.

2). Formación (docente o estudiantil) inadecuada o insuficiente para la enseñanza/aprendizaje de las matemáticas.

3). Papel que ejercen las actitudes, motivaciones, creencias y expectativas hacia las matemáticas.

Pero, el hecho de que los primeros cinco años de acercamiento a las matemáticas muestren un comportamiento decreciente, presupone que aumentarlo conlleva esfuerzo de estudiantes, docentes y familia. De acuerdo con Rivas (2019): la realidad familiar está identificada como uno de los factores más influyentes sobre el aprendizaje, junto a la motivación. Si no se le presta atención al desempeño en matemáticas, los problemas de las edades 
tempranas tienden a incrementarse progresivamente (pág. 1).

$\mathrm{Si}$ un comienzo como el que se muestra en la figura 1, lleva una tendencia a la estandarización, tendría incidencia en el aprendizaje secuencial $y$ progresivo en que se articulan los contenidos cognoscitivos y procedimentales que caracterizan el cambio curricular de Matemática. Cuando el estudiante va olvidando lo que aprendió en años anteriores (incluso operaciones básicas) o quedan conceptos por aprender o competencias por desarrollar, se dificultan los aprendizajes posteriores. También tiene consecuencias en la vida cotidiana, al establecerse las matemáticas como una competencia básica para desplegarse con acierto en la sociedad.

\section{4. ¿Cómo se puede mejorar?}

Se considera un objetivo en la enseñanza de las matemáticas mejorar la práctica de los estudiantes en la resolución de problemas. Esto constituye un reto:

1. Del docente exige un comportamiento creativo y flexible que va más allá de la adquisición de conocimientos. Lo cual lo ubica en el contexto tecnológico desafiante con diferentes perspectivas para hacer, ser y compartir.

2. Del estudiante, estar dispuesto a consumir energía mental y a esforzarse. El cerebro tiende a la economía cognitiva, mientras el lenguaje simbólico y abstracto de la matemática exige una actitud más activa.

A partir de la visualización de los datos generados por esta investigación se piensa en la aplicación de estrategias que contribuyan a evitar la fatiga mental; por ejemplo, la aplicación de ejercicios que fortalezcan la atención positiva del estudiante. Dichos ejercicios deben ajustar contextualmente la relación objeto-sujeto del conocimiento a la complejidad-edad, para que 
cumplan el cometido para lo cual se aplican.

Los docentes pueden iniciar una actividad que después sea mantenida por la motivación intrínseca, que provoque en el estudiante el deseo de aprender para cumplir sus propios objetivos. Otra manera sería, potenciar el aprendizaje creativo a partir de la contextualización del contenido de la materia de matemática. De este modo se trabaja en acciones y actividades que fortalezcan los aprendizajes con calidad, calidez y de manera productiva.

\section{5. ¿Qué cambios se necesitan para promover el mejoramiento escolar?}

El análisis de datos contribuye a responder preguntas como estas. El problema resulta en que es difícil pasar exitosamente del final de un año académico a un nuevo final exitoso, si en el intermedio no visualizamos y evaluamos si lo que estamos haciendo está funcionando y cómo lo está haciendo. Las calificaciones o notas, en particular, son un medio subutilizado y potencialmente poderoso para la mejora de la enseñanza. Si bien sirven para propósitos importantes, carecen de los comentarios precisos y oportunos para informar la toma de decisiones sobre mejoras de la instrucción de los maestros y el apoyo para la mejora de la calidad de la educación.

En el caso particular de esta investigación, la recopilación, visualización y análisis de datos tuvo como un objetivo específico capturar el aprendizaje y la comprensión de los estudiantes. Luego se utilizó para guiar la práctica docente, al permitir elegir entre una atención individual o grupal, y evaluar la efectividad de los enfoques elegidos en la toma de decisiones.

La aplicación exitosa del análisis de datos densos a la formación docente para aumentar el desempeño de los estudiantes, es un desafío interdisciplinario que requiere:

1. Fuente de datos validables que puedan abordar preguntas de interés.

2. Habilidad de analizar e interpretar la información contenida en los datos y detectar patrones importantes. 
Pero esto crea una interrogante en la comprensión de los datos: ¿El uso

de los resultados de desempeño estudiantil para guiar la mejora de los aprendizajes tiene algunas debilidades inherentes?

Parece razonable que los patrones de bajo rendimiento que se perciben en el récord académico, reflejen una combinación de la instrucción que recibieron los estudiantes y los hábitos particulares de esa cohorte. Entonces, ¿Cómo deben saber los docentes si el siguiente grupo de estudiantes tiene el mismo conjunto de necesidades que el anterior?

El proceso de indagar sobre el significado de la información contenida en los datos de desempeño académico se convierte en parte de la experiencia de formación docente y formación integral de estudiantes. El reto quedaría en hacerse partícipe de la cultura de datos para la toma de decisiones basadas en evidencias, tomando en consideración que el uso sistemático de datos requiere un compromiso en muchos niveles. Cuando se busca utilizar los datos de manera más efectiva se necesita disciplina, capacitación, tecnología y más tiempo.

Ser parte de la cultura de datos en temas educativos ecuatorianos, por es una ventaja que pocos aprovechan. Al estar incorporado al desarrollo tecnológico y este a una brecha determinada por el acceso, uso y apropiación de incluso los datos, lo que va a pasar entre la apropiación de la cultura de datos y su retraso para incorporarla es TIEMPO. Esta medida en la duración de los acontecimientos se refleja en la calidad de la educación y en el número de estudiantes que ven limitada la mejora de sus aprendizajes.

\section{Conclusiones}

Los datos que constantemente son generados en las instituciones educativas, pueden ser utilizados de manera más eficiente en la gestión de los aprendizajes. Sostener niveles adecuados de calidad educativa, desarrollar estrategias de mejora y contribuir a que los estudiantes obtengan mayores 
beneficios de la educación que reciben, dependen en gran medida de la capacidad de familiarización con la cultura de datos.

Los datos que se recopilan deben alinearse claramente con los objetivos de todo el sistema educativo. Es preciso garantizar que se pueda acceder a ellos con seguridad, analizarlos y compartirlos de manera responsable y eficiente entre los miembros de la comunidad educativa.

El análisis de datos ofrece condiciones para sostener niveles adecuados de calidad educativa. Muestra la posibilidad de utilización para mejorar los planes de estudio, medir el progreso hacia los objetivos de aprendizaje y desarrollar estándares de equidad.

Un reporte de desempeño grupal se perfila como una iniciativa para visibilizar a los padres de familia o representantes sobre la tendencia de logros por parte de los estudiantes. Sería suficiente con incluirlo en los informes académicos de los periodos de lectivos y poder monitorearlo.

Las necesidades, infraestructura y recursos de los cuales disponen las unidades educativas determinan los alcances y limitaciones para integrar el análisis de datos en las mejoras basadas en evidencia.

La cultura de datos contribuye a transformar el universo académico. Por una parte, los docentes pueden tomar decisiones informadas que incidan de manera positiva en los resultados de los estudiantes, y estos, a su vez, ganan conocimiento sobre lo que deben hacer para satisfacer sus necesidades académicas.

Se produce una cultura de datos cuando una institución educativa se compromete a usar datos para la mejora continua en los niveles de educación, y a su vez enfatiza en la colaboración y capacitación a los docentes para que tomen decisiones por las cuales serán responsables. 


\section{Referencias}

Bernhardt, V. (2000). Databases can help teachers with standards implementation. Monograph $\mathrm{n}^{\circ}$ 5. Oroville, C.A., United States: California Association for Supervision and Curriculum Development.

Boaler, J. (2016a,b,c,d). How you can be good at math, and other surprising facts about learning. [Vídeo]. New York, United States:

TED Conferences, LLC. Recovered from: https://youtu.be/3icoSeGqQtY Carrillo, N. (2016). El big data quiere mejorar la educación. España: Universitat Oberta de Catalunya. Recuperado de:

https://www.uoc.edu/portal/es/news/actualitat/2016/242-bigdataeducacion.html

Codding, J., \& Rothman, R. (1999). Just passing through: the life of an American high school. D. D. Marsh \& J. B. Codding (Eds.). The new American high school (pp. 3-17). Thousand Oaks, C.A., United States: Corwin Publishing.

Coughlin, M. (2014). Engaging Evidence: How Independent Colleges and Universities Use Data to Improve Student Learning. Washington, D.C., United States: Council of Independent Colleges. Recovered from: https://eric.ed.gov/?id=ED561079

Dougherty, C. (2015a,b). Use of Data to Support Teaching and Learning: A Case Study of Two School Districts. ACT Research Report Series, (1). Iowa City, United States: ACT, Inc. Recovered from: https://eric.ed.gov/?id=ED558033

Hernández, Y. (2018). Fomentando el aprendizaje por medio del Big Data. México: Inevery Crea México - SANTILLANA. Recuperado de: https://ineverycrea.mx/comunidad/ineverycreamexico/recurso/fomenta ndo-el-aprendizaje-por-medio-del-big-data/45099a8d-2997-9f22-1c5e$\underline{57 f 4 c 00 f 51 d 4}$

Millett, C., Payne, D., Dwyer, C., Stickler, L., \& Alexiou, J. (2008). A Culture of 
Evidence: An Evidence-Centered Approach to Accountability for Student Learning Outcomes. Princeton, N.J., United States: Educational Testing Service. Recovered from:

https://eric.ed.gov/?id=ED499994

Navarro-Aburto, B., Arriagada, I., Osse-Bustingorry, S., \& Burgos-Videla, C. (2016). Adaptaciones curriculares: Convergencias y divergencias de su implementación en el profesorado chileno. Revista Electrónica Educare, 20(1), 1-18, e-ISSN: 1409-4258. Recuperado de:

https://www.redalyc.org/articulo.oa?id=194143011015

Parra, V., \& Matus, G. (2016). Usos de datos y mejora escolar: Una aproximación a los sentidos y prácticas educativas subyacentes a los procesos de toma de decisiones. Calidad en la educación, (45), 207-250, e-ISSN: 0718-4565. Recuperado de:

https://dx.doi.org/10.4067/S0718-45652016000200007

Rivas, A. (2019). Factores que producen diferencias significativas en el aprendizaje de los estudiantes que egresan de sexto grado. Revista Scientific, 4(12), 28-46, e-ISSN: 2542-2987. Recuperado de:

https://doi.org/10.29394/Scientific.issn.2542-2987.2019.4.12.1.28-46

Zhou, X. (2012). Girls' verbal skills make them better at arithmetic. Washington, D.C., United States: Association for Psychological Science. Recovered from:

https://www.psychologicalscience.org/news/releases/girls-verbal-skillsmake-them-better-at-arithmetic.html 


\section{Melvis Lissety González Acosta \\ e-mail: melvis.gonzalez@unae.edu.ec}

Nacida en Azogues, Ecuador, el 27 de febrero del año

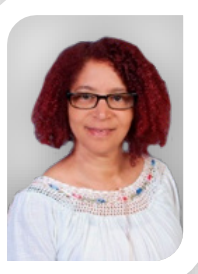
1973. Graduada de Filología por la Universidad Central de Las Villas (UCLV, 1995), Cuba; Magíster en Pedagogía por la Universidad Técnica Particular de Loja (UTPL, 2013), Ecuador; Doctora en Ciencias Sociológicas, mención en Desarrollo Social Comunitario por el Centro de Estudios Comunitarios de la (UCLV, 2017); Investigadora de campos sociales en línea; Indago sobre Gestión del conocimiento como recurso para el desarrollo global; Comparto el interés por la investigación interdisciplinaria con el trabajo de corrección de textos académicos y científicos; Escribo y edito el blog Miros Directory. 


\section{Diana Isabel Rodríguez Rodríguez \\ e-mail: diana.rodriguez@unae.edu.ec}

Nacida en Azogues, Ecuador, el 1 de mayo del año 1985.

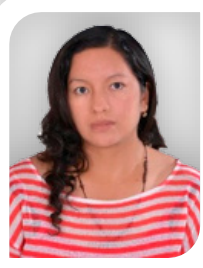

Graduada de Licenciada en Ciencias de la Educación

Mención: Matemática por la Universidad Tecnológica

Equinoccial de Quito (EHU, 2010), Ecuador; Magíster en

Gerencia y Liderazgo Educacional por la Universidad Técnica Particular de Loja (UTPL, 2016), Ecuador; Doctoranda en Ciencias de la Educación en la Universidad Nacional de Cuyo (UNCUYO), Mendoza, Argentina; Investigadora en Pedagogía de la Enseñanza de la Matemática y su interdisciplinaridad en abordaje de temas de interés para la educación actual. 